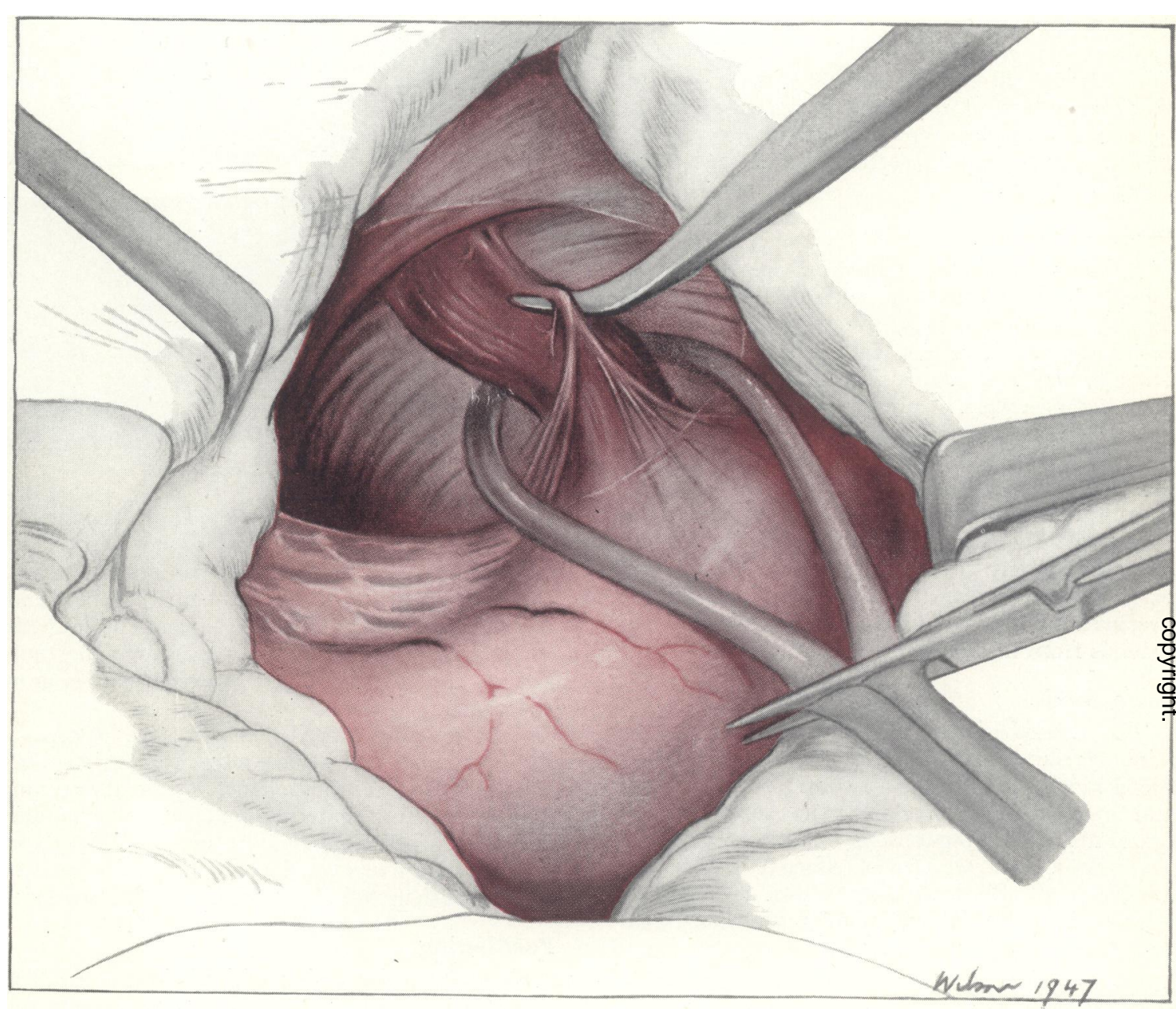

\title{
THE TECHNIQUE OF VAGAL RESECTION BY THE ABDOMINAL APPROACH
}

By IAN ORR, O.B.E., M.D., Ch.M., F.R.C.S.ED.

Department of Surgery, Postgraduate Medical School, London; Surgeon, Royal Infirmary, Preston

The operation of vagal resection in the treatment of peptic ulcer has received much prominence in America and the operative technique has been described by Dragstedt (1945-46). British surgeons have been cautious in their approach to the subject, and few references to the operative technique, its difficulties and drawbacks, have so far appeared in the literature in this country. The following description is based on the experience of 200 vagal resections performed by the author $\mathbb{D}$ and his colleague, Daintree Johnson, at the Postgraduate Medical School and other hospitals during the past five years. No attempt will be made to describe the rationale, the complications 
and the late results, as these have been fully reported in other journals (Orr and Johnson, 1947; Johnson, 1948; Orr, 1948). Combined procedures, such as vagotomy plus resection or gastro-enterostomy, and the indications for such combined procedures, will be described elsewhere. This article will confine itself to a description of the preparation of the patient, the steps in the operation and post-operative treatment, in the performance of vagal resection by the trans-hiatus route from below.

\section{Preparation}

As one of the immediate post-operative complications of vagotomy is patchy pulmonary collapse, it is wise to train the patient in selective breathing. If there is a history of chronic bronchitis or smokers' cough, it is advisable to cut out smoking for a week before operation, and the deep breathing exercises may, with advantage, be combined with penicillin inhalations.

On the morning of the operation, a Ryle's or Hamilton Bailey's tube is passed by the nasal route and the stomach emptied by aspiration. This tube is left in situ during the operation and early post-operative period. The abdomen is prepared up to the nipple line, as the incision extends from the xiphisternum.

\section{Anaesthetic}

The anaesthetic employed must provide for perfect relaxation and quiet breathing. Intercostal block, combined with continuous pentothal and cyclopropane; cyclopropane supported by one of the curare preparations, or continuous spinal anaesthesia have all proved effective. The disadvantage of the inter-costal block is the time taken to complete it, but lung complications seem to be less frequent with it.

\section{Instruments}

As the overhead shadowless lamp and spot light do not give sufficient illumination of the area below the diaphragm, a special illuminated retractor has been devised. The latest model, made for me by Messrs. Down Bros, and Mayer and Phelps, consists of a spoon-like blade with a reffecting surface on its concave aspect, and a serrated surface on the convex aspect. An electric bulb of the festoon type, fitted with a shade to prevent glare, throws a soft flood light over the field of the operation (Fig. I). The overhead shadowless light should be switched off when the illuminated retractor is in use, and if the theatre can be darkened, the illumination under the diaphragm is excellent.

A coldlite prostatic retractor is useful for exposing the under-surface of the oesophagus.
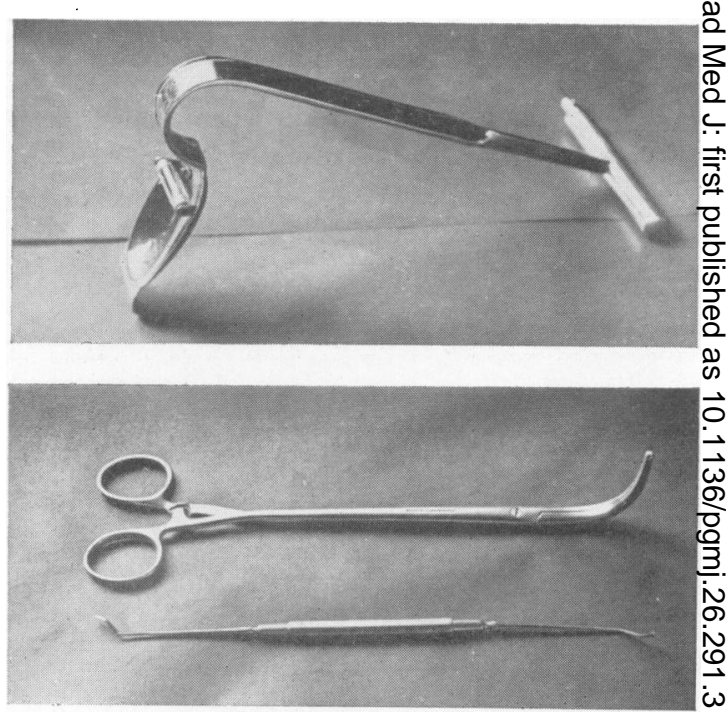

FIGS. 1 and 2

A dissector has been designed for the purposec of elevating the nerves from the oesophagus? (Fig. 2). It consists of a 9-in. handle, fenestrated to provide a firm grip, with a blade at either end, one curving to the right and one to the left. Bnce some dissections, one blade will prove the mae. serviceable; in others, the opposite blade is mere convenient.

A large, fully-curved clamp, of the pattern of Pigeon's forceps, is of value in introducing a rubber. tube around the oesophagus. The remaining instruments are of standard pattern-long-handled $\stackrel{2}{\overrightarrow{7}}$ curved scissors, Waugh's toothed dissecting for-윽 ceps, Moynihan's cholecystectomy forceps, or other long-handled curved forceps with fine jaws.

\section{Positioning}

The patient lies supine, with a bridge or sandbag: under the liver to open the infra-costal angle and 3 . provide better exposure. The anaesthetist mayo want to have both arms available for injection of $₹$ anaesthetic solutions, but these tend to get in theo way and if he can make use of the external jugular vein for his injection, the arms may be tuckedo down at the sides. The heels are supported on a rubber cushion, thereby removing all pressure or from the calves and lessening the chance of deep N vein thrombosis (Fig. 3).

\section{Technique of the Operation}

A mid-line incision commencing above the base $\stackrel{\circ}{\circ}$ of the xiphisternum and extending almost to the umbilicus, provides excellent exposure. The $\vec{\tau}$ stomach and duodenum are carefully inspected ${ }^{-}$ and the presence, position and size of the ulcer $\overrightarrow{\mathbb{D}}$ noted. The degree of patency and mobility of the $\frac{\Omega}{\mathbb{D}}$ 


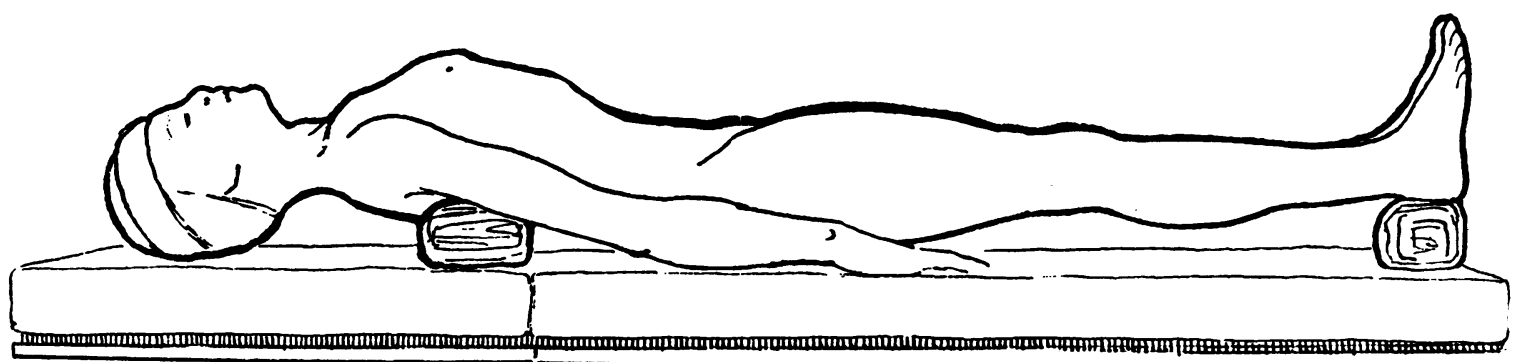

FIG. 3

pylorus is determined, and the final decision to perform vagotomy only, or to combine it with a resection or short-circuit is made. If the stomach is still distended with air, a sucker should be attached to the Ryle's tube and the organ completely deflated before proceeding to the next stage of the operation.

The left lobe of the liver lies over the oesophageal hiatus and must be mobilized and retracted. The left hand is introduced into the abdomen and the left lobe of the liver is grasped and drawn down. This puts the avascular triangular ligament on the stretch and it is then divided with curved scissors, held in the right hand. Occasionally, in deep-chested individuals, this step may be very difficult, and it may be found easier to grasp the liver with the right hand, using the second and third fingers to display the ligament, which is divided with a scalpel held in the left hand. The left phrenic vein is a potential danger at this stage. It should be visualized as it arches across the diaphragm, close to the attachment of the ligament, and great care should be taken to avoid wounding it. This accident happened on one occasion and very troublesome and profuse bleeding resulted.

When the left lobe of the liver is completely mobilized, it is folded down and covered by a sheet of rubber, being held aside by the special illuminated retractor. This instrument is held by the second assistant, standing on the left of the operator. The retractor must be held at such an angle that the light is reflected on to the oesophageal hiatus.

The exact position of the oesophagus may be determined by palpating the Ryle's tube as it enters the stomach. The peritoneum over this spot is picked up with Waugh's forceps and a small transverse incision made with long curved scissors (Fig. 4). It is usually easy to avoid damaging small vessels. As soon as the oesophagus is identified, the curved scissors are passed up through the hiatus and, by opening the scissors, the hiatus is defined and stretched. The forefinger of the right hand is then passed through the hiatus, then two fingers, and the oesophagus is gently mobilized. The forefinger is then passed behind it from left to right. Some difficulty may be experienced in penetrating the remnants of the dorsal mesentery, which anchors the oesophagus in the thorax, but by gentle dissection with the forefinger, a way through will be made, and the oesophagus can then be hooked downward and forward. It is wise at this juncture to determine whether one has hooked forward the posterior vagus nerve with the oesophagus. It is easily palpable like a taut piano wire against the elasticity of the oesophagus. As a rule the nerve lies in areolar tissue, overlying the aorta, and is not encountered at this stage, but if it should be palpated, the task of its identification is greatlyo simplified. Having hooked the oesophagus for ward with the forefinger, a large fully-curvedo forceps, such as Pigeon's forceps, is passed by the left hand behind the oesophagus from right to left. A fine rubber tube is grasped in the jaws of the forceps as they project on the left side of the oesophagus and the forceps is withdrawn pulling the tube with it (Frontispiece). The ends of the tube are clamped together, and it is used as a tractor to put the nerves on the stretch.

A search is now made for the anterior nerve. It may be seen as a pale streak, lying closely applied to the anterior surface of the oesophagus, or it may not be visible and must be identified by palpation. If traction is made on the rubber tube, the nerve is put on the stretch and feels like a wire. It is then lifted up on the curved dissector and clamped with cholecystectomy forceps. There may be one trunk only or there may be two, or even more, and while the most usual position is on the anterior surface of the oesophagus, coursing diagonally downward from left to right, one must be on guard against missing an abnormally placed nerve. It may lie so far to the right of the oesophagus that it may be mistaken for the posterior nerve. This error should become apparent, however, when the nerve is traced up through the hiatus.

A segment of nerve, 3 to $4 \mathrm{~cm}$. in length, is resected, cutting the nerve as high as possible. It is not necessary to tie the upper end, as bleeding 


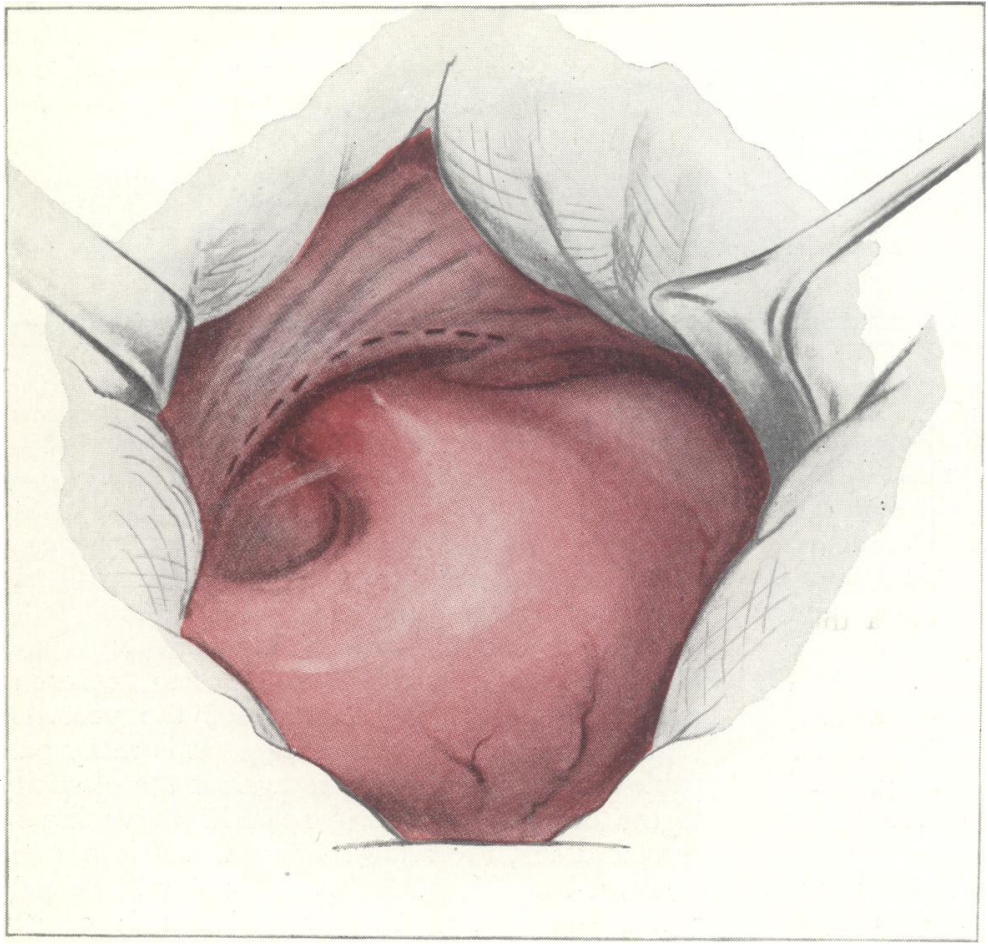

Fig. 4.-Incision of the peritoneunia

Fig. 5.-The posterior branch of the vagus.

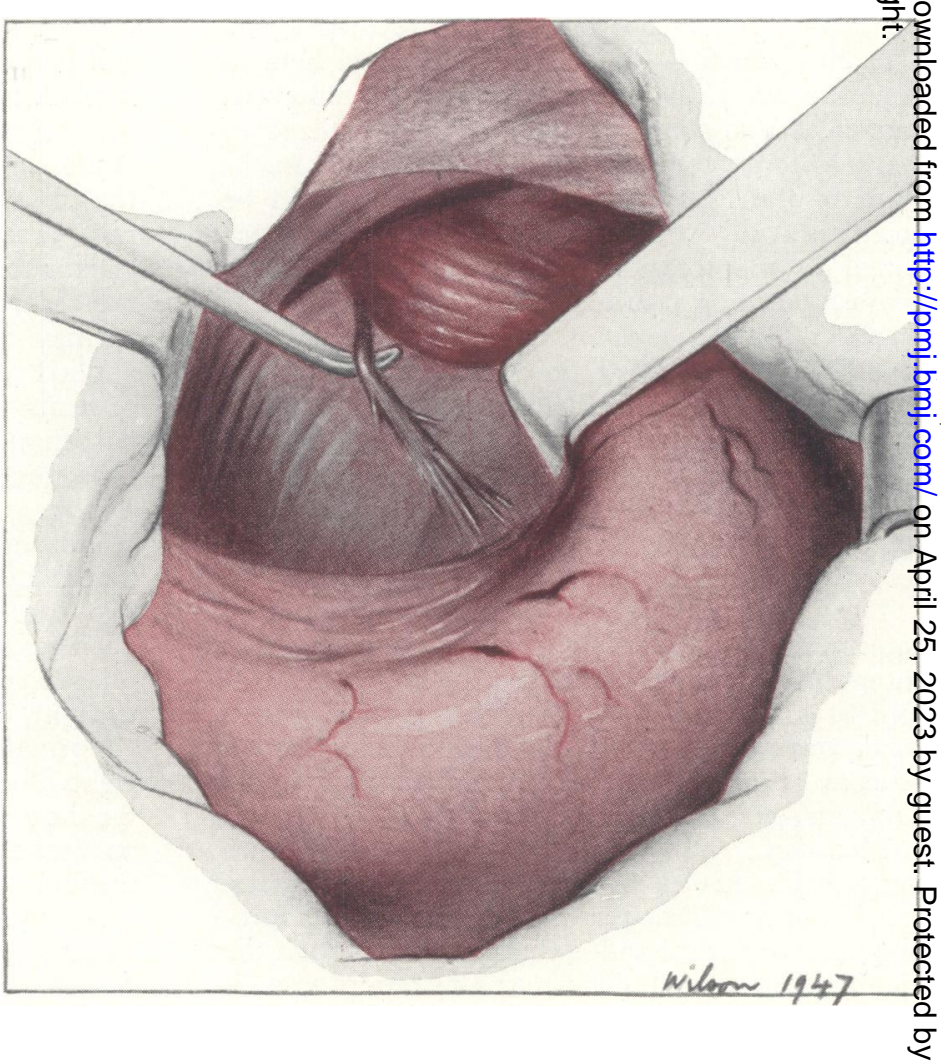


should not occur if the nerve has been properly identified and cleared. It is as well to ligature the lower cut end, however, as small blood vessels are frequently divided. Allen (1947) considers it advisable to cover the cut ends with a small silk capsule, to lessen the chances of regeneration.

Having dealt with the anterior nerve or nerves, the oesophagus is hooked to the left with the coldlite prostatic retractor and the illumination directed into the space between it and the aorta (Fig. 5). If, as is usual, the nerve lies in loose aerolar tissue overlying the aorta, it may not be easy to identify. It does not become taut, as does the anterior nerve, and indiscriminate dissection in the areolar tissue only results in obscuring the field by blood staining. A methodical plan must be followed if the nerve is to be found quickly. Firstly, before any palpating or dissecting is done, look carefully into the areolar tissue for a thick yellow streak, passing directly downwards through the hiatus. If this is seen, a curved dissector or the point of a cholecystectomy forcep may be used to elevate it. If this streak cannot be seen, the posterior surface of the oesophagus should be palpated again lest the nerve has been hooked forward. If not found, the nerve should be sought by gentle palpation, drawing the forefinger across the surface of the aorta till the right crus of the diaphragm is palpated. Very occasionally the nerve may be found closely applied to the right crus. A painstaking, methodical search is always rewarded by the identification of a thick cord. There is no doubt about it when it is found, and one should not be satisfied with less than a nerve $2 \mathrm{~mm}$. in diameter. Once found, traction should should be applied to it, to display branches or minor trunks. A segment of nerve is resected and the distal end ligated.

The rubber traction tube is now withdrawn and a final palpation with finger and thumb. is carried out to exclude the possibility of a minor branch being missed. The oesophagus is then allowed to fall back into the mediastinum and the peritoneum is closed over it with a few catgut sutures. The illuminated retractor is removed, the left lobe of the liver is allowed to fall back into position, and a final search for oozing is made before closing the abdomen. A most effective method of forestalling gastric retention is to coax the end of the Ryle's tube through the pylorus into the second part of the duodenum. This can be used for feeding the patient.

If additional operative procedures are considered necessary, they can be performed after closing the peritoneum over the oesophagus. There is always the risk of infecting the mediastinum if a resection or short-circuit is performed before carrying out the vagotomy.

\section{Post-Operative Treatment}

If an intravenous drip has not been set up $\frac{2}{3}$ during the operation, it is established as soon as $\stackrel{\mathbb{Q}}{\Omega}$ possible after the patient has returned to the ward. $c$ It is better to tie a cannula into the vein, rather than to rely on a needle remaining in position, as $\stackrel{\vec{D}}{\rightarrow}$ intravenous fluids are required for at least three days and often longer.

As soon as the patient is able to swallow, a second Ryle's tube is introduced through the other $\stackrel{\varnothing}{\varnothing}$ nostril and passed well into the stomach. This on is used for continuous suction and should be suitably labelled, to distinguish it from the tube in the duodenum. Suction is maintained by means of a motor or a Wangensteen apparatus. Daintree Johnson has designed a simple method of drip suction, which provides sufficient negative pressure to maintain a steady flow, without sucking o gastric mucosa into the holes in the tube.

A total of $2,000 \mathrm{ml}$. plus the quantity of fluid withdrawn by suction should be administered in $\omega$ the 24 hours. This is best given in the form of $\triangle$ $2,000 \mathrm{ml}$. of 5 per cent. dextrose in 0.16 per cent. saline, plus normal saline equal to the quantity of fluid aspirated. A check on urinary chlorides is kept daily, and if chlorides are in excess, the normal saline should be replaced by 0.16 per cent $\vec{\theta}$ saline.

Attempts to provide amino-acids intravenous in the form of casydrol proved disappointing, the casydrol appeared to precipitate venous thrombosis and the cannula had to be changed

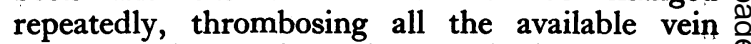
puncture sites and causing much discomfort to $\stackrel{\mathbb{Q}}{\Omega}$ the patient. The use of the indwelling duodenal $\vec{\overrightarrow{ }}$ tube has rendered the administration of intravenous castydrol unnecessary.

As soon as bowel movement can be detected by the stethoscope on the upper abdomen, ounce feeds of citrated milk and egg flip may be given 3 hourly through the duodenal tube. This commences, as a rule, 48 hours after the operation. If duodenal feeding is started too soon, a feeling of distention and epigastric discomfort ensues. Continuous gastric suction should be maintained, so long as a steady flow of fluid is aspirated. If there is little return, the suction may be disconnected and aspiration by a syringe carried out every two hours.

On the fourth day, a trial feed of $\mathrm{I}$ oz. of milk $N$ may be given and if aspiration two hours later $\underset{\omega}{N}$ shows that the milk has gone through, a 2-oz. O feed may be given, and if that has left the stomach in two hours, both gastric and duodenal tubes may be withdrawn and the patient allowed to $\stackrel{\odot}{\rightarrow}$ take water by mouth ad lib. and ounce feeds are 0 given hourly. A Ryle's tube should be passed at night, however, and the gastric contents aspirated, 
to ensure an empty stomach during the hours of sleep. If this evening aspiration fails to withdraw more than $100 \mathrm{cc}$. of gastric juice, it may be discontinued. If an increasing residue is found, particularly if milk has been retained, recourse to an indwelling tube and two-hourly suction may again be necessary.

In short, the aim of the post-operative regime is to decompress the stomach and give it no opportunity to over-distend, till there is evidence that tone has returned and it is capable of emptying itself. Failure to maintain gastric rest, during the period of gastric atony results in a dilatèd malfunctioning stomach, with all the unpleasant sequelae of belching and foul eructations.

Selective breathing exercises are recommenced as soon as the patient can co-operate. Frequent changes in position are encouraged and, as soon as the intravenous therapy is discontinued, the patient is allowed out of bed.

\section{Complications}

Partial Collapse of a Lung. This, unfortunately, is a common sequal to all vagotomy operations by the abdominal route. It appears to be less frequent in the patients anaesthetized by the intercostal block method and more frequent in those for whom curare has been used.

It has caused no serious anxiety in any patient and has always responded to penicillin therapy, combined with active postural manipulations. In no case has it been found necessary to aspirate the mucus plug with the bronchoscope:

Abdominal Distention. Severe and alarming distention has been noted in three patients, coming on 24 to 48 hours after operation. This responded to pituitrin injections.

Disruption of the Abdominal Wound. This occurred in three patients who had troublesome coughing after operation. Since the introduction of stainless steel wire for suturing the abdomen, it has not been noted.
Dysphagia. One patient complained of difỡ ficulty in swallowing. This persisted for severak weeks and a barium swallow revealed a condition akin to achalasia. This patient was one of a series.in which the lower end of the oesophagus was swabbed with 20 per cent. alcohol, with the object of destroying any small fibres missed at the operation. This step has been found unnecessary and potentially dangerous. The mild dysphagiaf occasionally encountered may be due to associated oesophagitis aggravated by the vagotomy.

Later Complications have been dealt with else $\overrightarrow{0}$ where (Orr, I948; Johnson, 1948).

There has been no death from vagotomy in this series. Convalescence has been rapid anḑ sedentary workers have been returned to work in a month to six weeks, and manual workers in two months after the operation.

\section{Follow-up Methods}

A complete and regular follow-up is essentiab in assessing the value of a new technique, and alt cases are seen at the follow-up clinics threen months, six months and one year after theik operation, and yearly thereafter. Those unable to attend are written to and a simple questionnaire is enclosed.

In order to compile data for future statistieal analysis, a punch-card system has been evolves on which are recorded all the more importan facts concerning the social history, sympto matology, investigation, type of operation and late results.

I am indebted to Messrs. Down Bros. and Messrs. Mayer and Phelps for their co-operation? and help in the design and manufacture of thes special instruments, and to Messrs. Copeland an Chatterson for advice and help in the preparingo of the follow-up card.

\section{BIBLIOGRAPHY}

ALLAN, A. W. (1947), Ann. R. Coll. Surgeons, Eng., I, 235.

DRAGSTEDT, L. R. (1945), Ann. Surg., 122, 973.

DRAGSTEDT, L. R. (1946), Minn. Med., 29, 597.

JOHNSON, H. D. (1948), Proc. Roy. Soc. Med., 4I, 649.

ORR, I. M. (1948), Proc. Roy. Soc. Med., 41, 639.

ORR, I. M., ard JOHNSON, H. D. (1947), Laniet, July 19, 84 